\title{
Transição epidemiológica nutricional em crianças e adolescentes argentinos de áreas carentes
}

\section{Nutritional epidemiological transition in poor Argentinean children and adolescents}

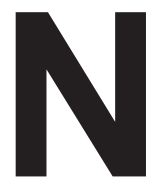

o Brasil, a maior parte dos estudos sobre nutrição realizada no século passado concentravase nos aspectos da desnutrição. Porém, dados mais recentes mostram redução da desnutrição e aumento de sobrepeso e obesidade em crianças, situação de "transição epidemiológica nutricional" que se caracteriza por diminuição de doenças transmissíveis e aumento das doenças crônicas não transmissíveis ${ }^{(1)}$. Nesse contexto, compartilham o mesmo cenário dois extremos da má nutrição: desnutrição pela carência e obesidade pelo excesso, o que se pode chamar de paradoxo nutricional ${ }^{(2)}$. Portanto, a obesidade e o sobrepeso constituem, na atualidade, importantes problemas de Saúde Pública, pelas elevadas taxas de prevalência, não somente em adultos, mas principalmente em crianças e adolescentes brasileiros ${ }^{(3-5)}$. O excesso de peso soma-se, em alguns casos, à dislipidemia e à hipertensão arterial como fatores de risco para o aparecimento de eventos cardiovasculares ${ }^{(6,7)}$.

Fatores externos socioambientais têm sido considerados de maior relevância na incidência de obesidade do que os fatores genéticos. Dentre os principais fatores externos relacionados ao desenvolvimento da obesidade, destacam-se a exposição prolongada à escassez de alimentos - intra ou extrauterina - levando à desnutrição e tendência à obesidade posteriormente, a transição nutricional com a troca do padrão tradicional para o contemporâneo (preferência por alimentos industrializados) e o estilo de vida urbano, marcado pelo sedentarismo da população. Consequências importantes dessas mudanças são as doenças associadas ao excesso de peso ${ }^{(8)}$. Aterosclerose e hipertensão arterial, doenças típicas de adultos, são processos iniciados na infância e relacionados à obesidade. Na atualidade, não há dúvida de que uma abordagem preventiva deve ser iniciada já na infância e adolescência. Além de ser uma intervenção benéfica, é na infância que são formados os hábitos alimentares e de estilo de vida saudável com redução do sedentarismo e estimulo à atividade física ${ }^{(8,9)}$.

Na América Latina, a prevalência de desnutrição crônica tem tendência decrescente: passou de $25,6 \%$ em 1980 para $12,6 \%$ em $2000^{(10)}$. No outro extremo das desordens nutricionais, encontra-se o excesso de peso, especificamente o sobrepeso e a obesidade, que constituem problemas de Saúde Pública classificados como doenças crônicas não transmissíveis. Sua frequência atinge de forma significativa a população argentina e, segundo um estudo realizado entre 1998 e 2001 , o sobrepeso ocorre em $20,8 \%$ e a obesidade em $5,4 \%$. Resultado semelhante é observado em outros países latino-americanos ${ }^{(11,12)}$. O fenômeno da Transição Epidemiológica Nutricional tem sido constatado em diversos estudos realizados na América Latina, nas últimas décadas. Como acontece no Brasil, o mesmo se verifica na Argentina, onde a transição epidemiológica nutricional em alguns grupos populacionais é cada vez mais frequente. Assim, pode-se observar a coexistência de problemas nutricionais tanto pelo déficit como pelo excesso de peso ${ }^{(13,14)}$.

Pais e Carrera, da Universidad Nacional del Litoral, em Santa Fé, Argentina, avaliaram o estado nutricional de crianças e adolescentes moradoras de áreas carentes para detectar alterações nutricionais e de crescimento e possíveis fatores de risco para eventos cardiovasculares na idade adulta(15). Os autores avaliaram 136 crianças e adolescentes (49\% do sexo feminino), com idade média de 10,0 $\pm 2,6$ anos e variação de 5 a 15 anos. Do total de crianças, $85 \%$ passavam mais de três horas por dia em frente à televisão e $25 \%$ não realizavam atividades físicas. Do grupo analisado, $14,7 \%$ eram desnutridos agudos, sendo $7,5 \%$ na forma crônica. O sobrepeso estava presente em $11,7 \%$ e a obesidade em $2,9 \%$. A comparação desses valores com os de prevalência nacional argentina de desnutrição crônica (12\%) não mostrou diferença significativa. Entre os desnutridos agudos, observouse uma taxa de $14 \%$, muito superior aos dados nacionais (frequência máxima de $3 \%$ : $p=0,002$ ). Quanto aos dados clínicos, do total de crianças e adolescentes analisados, 26 (20\%) apresentaram valores sugestivos de hipertensão. Desses, quatro eram obesos e cinco desnutridos. Quanto ao sexo, 17 eram do sexo masculino e 9 do sexo feminino $(p=0,025)$. Comparou-se a frequência de crianças com sobrepeso e obesidade com hipertensão (4/20) à frequência de desnutridos com pressão arterial acima do percentil 95 (5/30), ha- 
vendo similaridade $(p=0,99)$. Os resultados quanto à pressão arterial permitem inferir uma prevalência de hipertensão de $20 \%$, sendo $10 \%$ maior do que revelam os estudos realizados na Argentina. O perfil lipídico mostrou-se alterado em $49 \%$ da amostrar avaliada. Do grupo com alterações lipídicas, $22 \%$ tinham aumento de colesterol total, $12 \%$ LDL-C elevado e $15 \%$ valores de triglicérides alterados. A proporção de crianças e adolescentes com colesterol total, LDL-C e níveis de pressão arterial elevados mostrou que a dislipidemia aumentava 2,6 vezes a chance de risco de hipertensão arterial (Odds Ratio: 2,63; intervalo de confiança 95\%: 1,07-6,46). Das crianças com hipertensão arterial, nove tinham também níveis de colesterol superior aos valores normais. A alteração do perfil lipídico aumentou a chance de hipertensão arterial. O risco de hipertensão foi 3,6 vezes maior para o sexo feminino e aumentou 2,5 vezes para cada unidade de aumento do LDL-C/HDL-C. Somados a esses achados clínicos e laboratoriais, os hábitos de vida contribuíram mais ainda para os fatores de risco.

No estudo Bogalusa, em crianças americanas com sobrepeso e obesidade, o colesterol total foi 2,4 a 7,1 vezes mais elevado, enquanto o LDL-C foi 12,6 vezes maior. O LDL colesterol e o IMC foram os fatores mais fortemente relacionados ao aumento de espessura da camada íntima arterial(16,17).

Em Belo Horizonte, um estudo com escolares investigou associação do sobrepeso e da obesidade com pressão arterial, lipídeos séricos e atividade física. Crianças e adolescentes com sobrepeso ou obesidade, assim como os sedentários, apresentaram níveis mais elevados de pressão arterial e perfil lipídico de risco para o desenvolvimento da aterosclerose e síndrome metabólica ${ }^{(18)}$. A cada cinco escolares, $19,3 \%$ apresentaram quatro fatores de risco concomitantes: nível elevado de colesterol total (>200mg/dL), IMC > P85, pressão arterial sistólica > P90, pressão arterial diastólica > P90.

Um achado interessante foi a ausência de anemia na amostra estudada. Estudos anteriores na mes- ma região revelam elevada prevalência de anemia ${ }^{(19)}$. Ações de uma Organização Não Governamental que presta assistência e distribui alimentos à população ajudam a explicar esse achado. A oferta de alimentos com adequado conteúdo de ferro pode ter contribuído para a menor prevalência de anemia. Nenhum dos participantes tinha níveis de hemoglobina sugestivos de anemia, porém, não se determinaram os valores de ferro sérico e outros parâmetros para avaliar o estado nutricional de ferro, sem dúvida uma das principias carências nutricionais e um problema de Saúde Pública ainda não controlado na América Latina e no Brasil(20).

Nutrição está se tornando o mais modificável determinante das doenças crônicas. O sobrepeso e a obesidade têm um impacto significante na saúde física e psicossocial, requerendo, portanto, estratégias de prevenção e controle. A dieta exerce forte efeito positivo ou negativo sobre a saúde e pode influir sobre riscos de morbidade em curto prazo, com impacto significativo sobre a qualidade de vida em longo prazo. A prevenção de doenças do adulto deve ser uma preocupação do pediatra já na infância. Do ponto de vista de Saúde Pública, em países em desenvolvimento, o combate à fome se justifica visto que ainda existem bolsões de pobreza com desnutrição. Entretanto, outra importante questão é a distribuição de renda, em geral concentrada em grande parte nas mãos de uma pequena parcela da população. Esses são fatores que devem ser considerados em análise mais profunda da questão nutricional e de suas implicações na saúde das populações brasileira e argentina. Assim como no Brasil, tanto a fome zero quanto a obesidade zero deveriam ser parte de uma mesma política, compreendendo um programa de educação nutricional e melhor distribuição de renda para que as famílias possuam maior conhecimento e tenham mais acesso aos alimentos de qualidade nutricional. Nesse contexto, o artigo aqui publicado contribui para o melhor conhecimento da situação nutricional em crianças e adolescentes em áreas carentes da Argentina.

Joel Alves Lamounier ${ }^{1}$ 


\section{Referências bibliográficas}

1. Batista Filho M, Rissin A. A transição nutricional no Brasil: tendências regionais e temporais. Cad Saude Publica 2003;19 (Suppl 1):S181-91.

2. Vieira EC, Leão E, Lamounier JA. Desnutrição versus obesidade: o paradoxo nutricional no Brasil. Rev Med Minas Gerais 2004;14:1.

3. Cintra IP, Passos MA, Fisberg M, Machado HC. Evolução de duas séries históricas do índice de massa corporal em adolescentes. J Pediatr (Rio J) 2007;83:157-62.

4. IBGE - Instituto Brasileiro de Geografia e Estatística. Pesquisa de orçamentos familiares 2002-2003: antropometria e análise nutricional de crianças e adolescentes no Brasil. Rio de Janeiro: IBGE; 2006.

5. Lamounier JA, Abrantes MM, Colosimo EA. Prevalência de sobrepeso e obesidade em crianças e adolescentes das regiões Sudeste e Nordeste. J Pediatr (Rio J) 2002;78:335-40.

6. Dirisamer A, Widhalm K. Lipoprotein (a) as a potent risk indicator for early cardiovascular disease. Acta Paediatr 2002;91:1313-7.

7. Wiegman A, Rodenburg J, de Jongh S, Defesche JC, Bakker HD, Kastelein $\mathrm{JJ}$ et al. Family history and cardiovascular risk in familial hypercholesterolemia: data in more than 1000 children. Circulation 2003;107: 1473-8.

8. Parizzi MR, Cunha CF, Ferreira RA, Lamounier JA, Listgarten MA. Abordagem interdisciplinar do adolescente obeso com ênfase nos aspectos psicossociais e nutricionais. Rev Med Minas Gerais 2008;18: 154-60.

9. Lamounier JA, Diamante R, Parizzi MR, Rossetti MB, Ribeiro RQ. Obesidade e dislipidemias. Programa Nacional de Educação Continuada em Pediatria da Sociedade Brasileira de Pediatria, PRONAP 2006;IX: 23-70.

10. de Onis M, Frongillo EA, Blössner M. Is malnutrition declining? An analysis of changes in levels of child malnutrition since 1980. Bull World Health Organ 2000;78:1222-33.
11. Kovalskys I, Bay L, Herscovici CR, Berner E. Prevalencia de obesidad en la población de 10 a 19 años en la consulta pediátrica. Arch Argent Pediatr 2003;101:441-7.

12. Lobstein T, Baur L, Uauy R; IASO International Obesity Task Force. Obesity in children and young children: a crisis in public health. Obes Rev 2004;5 (Suppl 1):4-104.

13. Durán P. Transición epidemiológica nutricional o el efecto mariposa. Arch Argent Pediatr 2005;103:195-7.

14. Orden AB, Torres MF, Luis MA, Cesani MF, Quintero FA, Oyhenart EE. Evaluación del estado nutricional en escolares de bajos recursos socioeconómicos en el contexto de la transición nutricional. Arch Argent Pediatr 2005;103:205-11.

15. Pais AB, Carrera EF. Necessidades básicas insatisfeitas, desnutrição infantil e risco de doenças na vida. Rev Paul Pediatr 2009;27:127-32.

16. Freedman DS, Dietz WH, Srinivasan SR, Berenson GS. The relation of overweight to cardiovascular risk factors among children and adolescents: The Bogalusa Heart Study. Pediatrics 1999;103:1175-82.

17. Li S, Chen W, Srinivasan SR, Bond MG, Tang R, Urbina EM et al. Childhood cardiovascular risk factors and carotid vascular changes in adulthood: the Bogalusa Heart Study. JAMA 2003;290:2271-6.

18. Ribeiro RQ, Lotufo PA, Lamounier JA, Oliveira RG, Soares JF, Botter DA. Fatores adicionais de risco cardiovascular associados ao excesso de peso em crianças e adolescentes. O estudo do coração de Belo Horizonte. Arq Bras Cardiol 2006;86:406-16.

19. Argentina. Ministerio de Salud. Presidencia de la Nación. Encuesta Nacional de Nutrición y Salud [homepage on the Internet]. Documento de Resultados 2007 [cited 2008, Mai 20]. Available from: http://www.msal.gov.ar/htm/site/ ennys/pdf/documento_resultados_2007.pdf

20. Rocha DR, Lamounier JA, Capanema FD, Franceschini SC, Norton RC, Costa $\mathrm{AB}$ et al. Estado nutricional e anemia de crianças que freqüentam creches em Belo Horizonte, Minas Gerais. Rev Paul Pediatr 2008;26:6-13.
Instituição: Departamento de Pediatria da Universidade Federal de Minas Gerais (UFMG), Belo Horizonte, MG, Brasil

${ }^{1}$ Professor titular de Pediatria da Faculdade de Medicina da UFMG, Belo Horizonte, MG, Brasil
Endereço para correspondência:

Joel Alves Lamounier

Faculdade de Medicina da UFMG

Avenida Alfredo Balena, 190

CEP 30130-100 - Belo Horizonte/MG

E-mail: jalamo@medicina.ufmg.br 